PROCEEDINGS OF THE

AMERICAN MATHEMATICAL SOCIETY

Volume 129 , Number 10, Pages 3091-3100

S 0002-9939(01)05824-5

Article electronically published on May 10, 2001

\title{
UNIFORMLY MORE POWERFUL TESTS FOR HYPOTHESES ABOUT LINEAR INEQUALITIES WHEN THE VARIANCE IS UNKNOWN
}

\author{
YINING WANG AND MICHAEL P. MCDERMOTT \\ (Communicated by Wei Y. Loh)
}

\begin{abstract}
Let $\mathbf{X}$ be a $p$-dimensional normal random vector with unknown mean $\mu$ and covariance matrix $\Sigma=\sigma^{2} \Sigma_{0}$, where $\Sigma_{0}$ is a known matrix and $\sigma^{2}$ an unknown parameter. This paper gives a test for the null hypothesis that $\mu$ lies either on the boundary or in the exterior of a closed, convex polyhedral cone versus the alternative hypothesis that $\mu$ lies in the interior of the cone. Our test is uniformly more powerful than the likelihood ratio test.
\end{abstract}

\section{INTRODUCTION}

Let $\mathbf{X}=\left(X_{1}, \ldots, X_{p}\right)^{\prime}, p \geq 2$, be a $p$-dimensional normal random vector with unknown mean $\mu=\left(\mu_{1}, \ldots, \mu_{p}\right)^{\prime}$ and covariance matrix $\Sigma=\sigma^{2} \Sigma_{0}$, where $\Sigma_{0}$ is a known matrix. Let $b_{1}, \ldots, b_{m}$ be $m(m \geq 2)$ specified $p$-dimensional vectors and consider the problem of testing

$$
H_{0}^{*}: \min _{1 \leq i \leq m} b_{i}^{\prime} \mu \leq 0 \text { versus } H_{1}^{*}: \min _{1 \leq i \leq m} b_{i}^{\prime} \mu>0,
$$

assuming that $\left\{b_{1}, \ldots, b_{m}\right\}$ is without positive relations and has no redundant vectors (Sasabuchi [7]). This is equivalent to testing the null hypothesis that $\mu$ lies either on the boundary or in the exterior of a closed, convex polyhedral cone versus the alternative hypothesis that $\mu$ lies in the interior of the cone. Common applications of the testing problem (1.1) arise in the setting of equivalence testing (Berger and Hsu [2]) and clinical trials of combination therapies (Laska and Meisner [3]).

Sasabuchi ([7], 8]) derived the likelihood ratio test (LRT) for a problem very similar to (1.1), and Berger [1] showed that this test was also the LRT for (1.1). The LRT is biased, and the level $\alpha$ is attained only in the limit if one $b_{i}^{\prime} \mu=0$ and $b_{j}^{\prime} \mu \rightarrow \infty$ for all $j \neq i$ (Sasabuchi [7, 8]). Berger [1, Liu and Berger [4], and McDermott and Wang [6] constructed classes of size- $\alpha$ tests that are uniformly more powerful than the LRT for the case of $\sigma^{2}$ known. Berger 1] also demonstrated that his approach does not yield a size- $\alpha$ test for the case of $\sigma^{2}$ unknown.

In view of Liu and Berger [4, it is clear that when $m=2$, without loss of generality, the problem can be transformed to that of observing $(U, V)^{\prime} \sim$

Received by the editors May 13, 1998 and, in revised form, December 14, 1999 .

2000 Mathematics Subject Classification. Primary 62F03; Secondary 62F04, $62 \mathrm{H} 15$.

Key words and phrases. Conditional distribution, likelihood ratio test, one-sided testing, polyhedral cone. 
$N_{2}\left(\left(\mu_{1}, \mu_{2}\right)^{\prime}, \sigma^{2} I\right)$ and testing

$$
H_{0}: \min \left\{\mu_{1}+r \mu_{2}, \mu_{2}\right\} \leq 0 \text { versus } H_{1}: \min \left\{\mu_{1}+r \mu_{2}, \mu_{2}\right\}>0,
$$

with $-\infty<r<\infty$. In this paper, an approach based on conditioning arguments is used to construct tests for (1.2) that are uniformly more powerful than the LRT for the case of $\sigma^{2}$ unknown. This approach to test construction can be extended to the general problem (1.1), that is, $m \geq 2$ and $\Sigma_{0} \neq I$, by linear transformation and by applying the intersection-union method of Liu and Berger [4].

Throughout the paper, $P_{\left(\mu_{1}, \mu_{2}\right)}(\cdot)$ will be used to denote the probability measure with the indicated means and variance-covariance matrix $I$.

\section{Derivation of TESTS UNIFORMLy MORE POWERFUl THAN THE LRT}

In this section, an approach based on conditioning is used to derive tests that are uniformly more powerful than the LRT assuming $\Sigma=\sigma^{2} I$, with $\sigma^{2}$ unknown. Let $\mathcal{C}=\{(U, V): U+r V \geq 0, V \geq 0\}$. The following theorem due to Berger [1] is important in our derivation.

Theorem 2.1. Under $H_{0}$, for any fixed $\sigma^{2}>0$ and $A \subset \mathcal{C}$,

$$
\sup _{\mu_{1}, \mu_{2}} P_{\left(\mu_{1}, \mu_{2}\right)}(A)=\max \left\{\sup _{\mu_{1} \geq 0} P_{\left(\mu_{1}, 0\right)}(A), \sup _{\mu_{2} \geq 0} P_{\left(-r \mu_{2}, \mu_{2}\right)}(A)\right\} \text {. }
$$

Let $S^{2}$ be a random variable independent of $(U, V)$ such that $S^{2} / \sigma^{2}$ has a $\chi^{2}$ distribution with $\nu$ degrees of freedom, and let $W^{2}=S^{2}+U^{2}+V^{2}$. Also, let $T$ be a random variable such that $\sqrt{\nu} T$ has a $t$-distribution with $\nu$ degrees of freedom. For $\alpha<0.5$, Sasabuchi $([7,[8])$ showed that the critical region of the LRT is

$$
A_{0} \equiv\left\{(U, V, S):(U+r V) /\left(S \sqrt{1+r^{2}}\right) \geq t, V / S \geq t\right\}
$$

where $t$ is the upper $100 \alpha$ percentile of the distribution of $T$. Note that $t>0$, since $\alpha<0.5$. In view of Theorem 2.1, any test with critical region $R \subset \mathcal{C}$ has size $\alpha$ if and only if the following is satisfied:

$$
\alpha=\max \left\{\sup _{\mu_{1} \geq 0, \sigma^{2}>0} P_{\left(\mu_{1}, 0 ; \sigma^{2}\right)}[(U, V) \in R], \sup _{\mu_{2} \geq 0, \sigma^{2}>0} P_{\left(-r \mu_{2}, \mu_{2} ; \sigma^{2}\right)}[(U, V) \in R]\right\},
$$

where the probability measure now depends on the additional parameter $\sigma^{2}$ in the obvious way. Let

$$
X=\frac{U+r V}{\sqrt{1+r^{2}}}, \quad Y=\frac{-r U+V}{\sqrt{1+r^{2}}}, \quad \nu_{1}=\frac{\mu_{1}+r \mu_{2}}{\sqrt{1+r^{2}}}, \quad \nu_{2}=\frac{-r \mu_{1}+\mu_{2}}{\sqrt{1+r^{2}}} .
$$

Then $X \sim N\left(\nu_{1}, \sigma^{2}\right), Y \sim N\left(\nu_{2}, \sigma^{2}\right)$, and $X, Y$, and $S^{2}$ are mutually independent. Thus

$$
\sup _{\mu_{2} \geq 0, \sigma^{2}>0} P_{\left(-r \mu_{2}, \mu_{2} ; \sigma^{2}\right)}[(U, V) \in R]=\sup _{\nu_{2} \geq 0, \sigma^{2}>0} P_{\left(0, \nu_{2} ; \sigma^{2}\right)}\left[(X, Y) \in R^{\prime}\right],
$$

where $R^{\prime}$ is the appropriately transformed critical region.

Note that $A_{0}$, the critical region for the LRT, satisfies (2.2) because the following inequalities hold for every $u, y$, and $w$, the observed values of $U, Y$, and $W$, 
respectively:

$$
\begin{aligned}
& P_{\left(\mu_{1}, 0\right)}\left((U+r V) /\left(S \sqrt{1+r^{2}}\right) \geq t, V / S \geq t \mid U=u, W=w\right) \leq \alpha, \\
& P_{\left(0, \nu_{2}\right)}\left((r X+Y) /\left(S \sqrt{1+r^{2}}\right) \geq t, X / S \geq t \mid Y=y, W=w\right) \leq \alpha .
\end{aligned}
$$

Because the above inequalities are strict for some values of $u, y$, and $w$, the rejection region of the LRT may be expanded while preserving the size of the test to be less than or equal to $\alpha$. The general approach that follows is similar to that of McDermott and Wang [6], but with further conditioning on $W$ to deal with the nuisance parameter $\sigma^{2}$.

2.1 Derivation for $r \leq 0$. Denote $\gamma=|r|$. The critical region of the LRT is

$$
A_{0}=\left\{(U, V, S):(U-\gamma V) /\left(S \sqrt{1+\gamma^{2}}\right)>t, V / S>t\right\} .
$$

Let $\left(u^{*}, v^{*}\right)$ be the solution to the system

$$
\left\{\begin{array}{l}
u^{2}+v^{2}=w^{2} \\
u-\gamma v=0
\end{array}\right.
$$

Then $v^{*}=w / \sqrt{1+\gamma^{2}}$ and $u^{*}=\gamma v^{*}$ (see Figures 1 and 2).

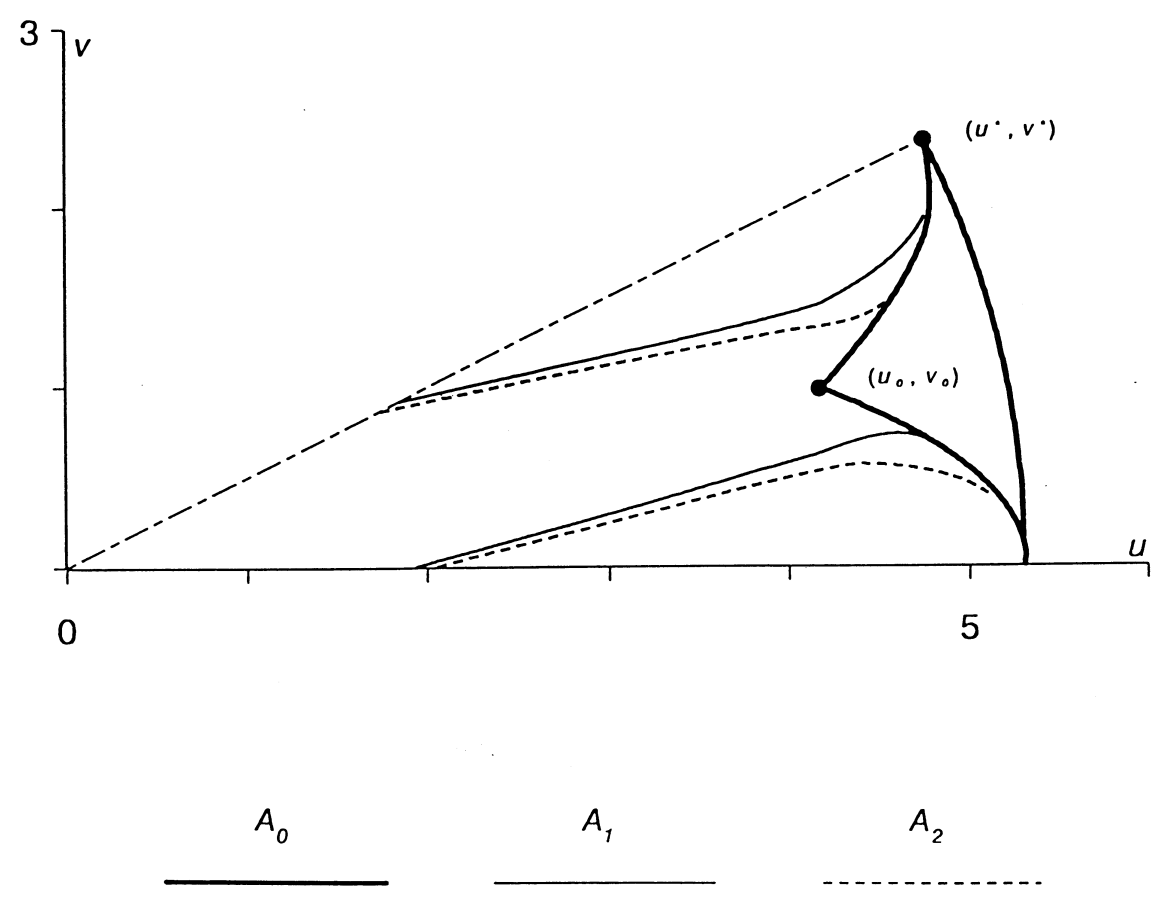

FiguRE 1. Illustration of $A_{0} \cup\left(A_{1} \cap A_{2}\right)$, the critical region of the uniformly more powerful test for $r<0$ and $u_{0}<u^{*}$, conditional on $W=w$. The values used for computation were $\alpha=0.20, \nu=8$, $r=-2$, and $w^{2}=28.2$. 

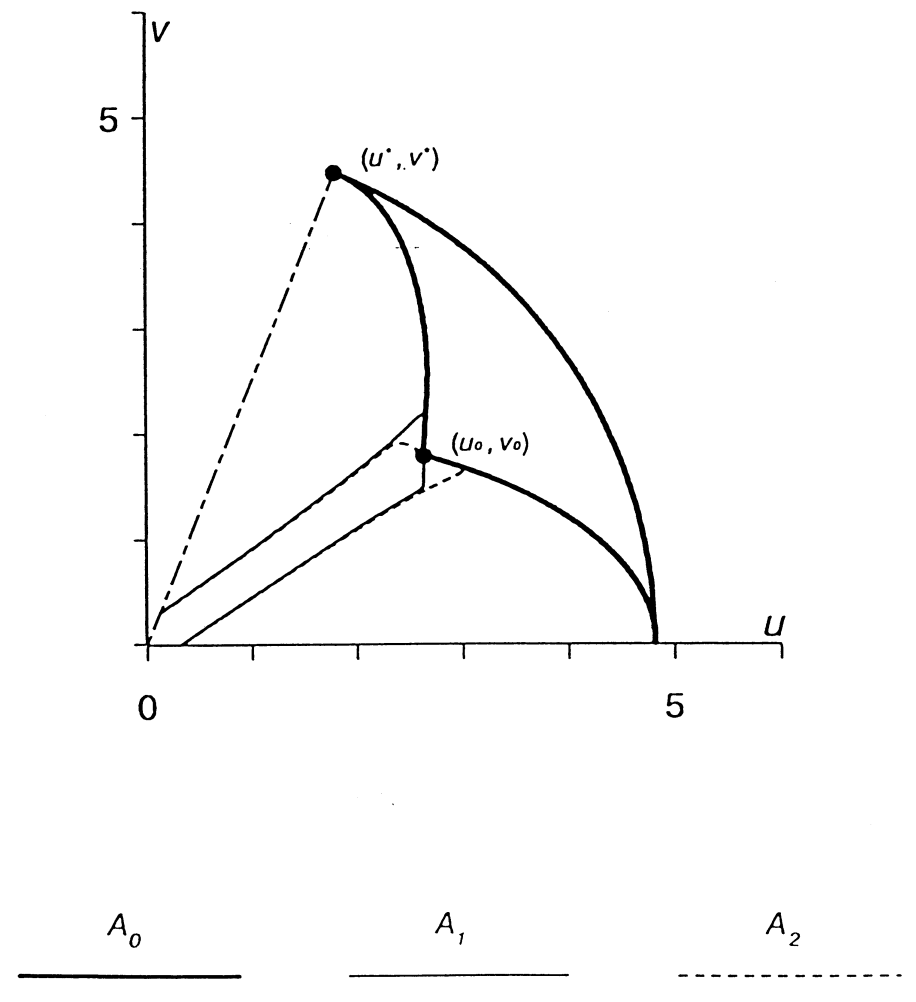

Figure 2. Illustration of $A_{0} \cup\left(A_{1} \cap A_{2}\right)$, the critical region of the uniformly more powerful test for $r<0$ and $u_{0}>u^{*}$, conditional on $W=w$. The values used for computation were $\alpha=0.10, \nu=8$, $r=-0.4$, and $w^{2}=23.2$.

Let $\left(u_{0}, v_{0}\right)$ be the intersection point of the curves $(u-\gamma v) /\left(\sqrt{1+\gamma^{2}} s\right)=t$ and $v / s=t$, which are the boundary curves of $A_{0}$ (see Figures 1 and 2). That is, $\left(u_{0}, v_{0}\right)$ is the solution to the system

$$
\left\{\begin{array}{l}
(u-\gamma v) / \sqrt{\left(1+\gamma^{2}\right)\left(w^{2}-u^{2}-v^{2}\right)}=t, \\
v / \sqrt{w^{2}-u^{2}-v^{2}}=t
\end{array}\right.
$$

subject to $v>0$ and $v \gamma<u$. The solution is

$$
v_{0}=t w / \sqrt{1+t^{2}+t^{2}\left(\gamma+\sqrt{1+\gamma^{2}}\right)^{2}}, \quad u_{0}=\left(\gamma+\sqrt{1+\gamma^{2}}\right) v_{0} .
$$

2.1.1 The case of $u_{0} \leq u^{*}$. It can be easily verified that $A_{0}$ is contained in the positive orthant. Note also that

$$
\begin{aligned}
& \left\{(U, V, S): V>0, U \leq u^{*}, U-\gamma V>0,(U-\gamma V) / S>\sqrt{1+\gamma^{2}} t\right\} \\
= & \left.(U, V, S): V>0, \gamma V<U \leq u^{*},(U-\gamma V)^{2}>\left(1+\gamma^{2}\right) t^{2}\left(W^{2}-U^{2}-V^{2}\right)\right\} \\
= & \left\{(U, V, S): V>0, \gamma V<U \leq u^{*},\right. \\
& \left.V<\left[\gamma U-\sqrt{d\left(1+\gamma^{2}\right) t^{2}\left(W^{2}-U^{2}\right)+\left(\gamma^{2}-d\right) U^{2}}\right] / d\right\},
\end{aligned}
$$


where $d=t^{2}+\gamma^{2}+\gamma^{2} t^{2}$. The last equation in (2.6) holds because the critical region is under the curve $v=\gamma u / d-\sqrt{d\left(1+\gamma^{2}\right) t^{2}\left(w^{2}-u^{2}\right)-d u^{2}+\gamma^{2} u^{2}} / d$ (see Figure 1). Now define, for $u \leq u^{*}$,

$$
g(u, w)=\gamma u / d-\sqrt{d\left(1+\gamma^{2}\right) t^{2}\left(w^{2}-u^{2}\right)-d u^{2}+\gamma^{2} u^{2}} / d .
$$

From (2.5) and (2.6), it follows that

$$
A_{0} \cap\left\{(U, V, S): U \leq u^{*}\right\}=\left\{(U, V, S): U \leq u^{*}, t S<V<g(U, W)\right\} .
$$

Consequently, for $u \leq u^{*}$,

$$
\begin{aligned}
P_{\left(\mu_{1}, 0\right)} & \left(A_{0} \mid U=u, W=w\right) \\
= & \begin{cases}0 & \text { if } u<u_{0}, \\
P_{\left(\mu_{1}, 0\right)}[t S<V<g(U, W) \mid U=u, W=w] & \text { if } u_{0} \leq u \leq u^{*} .\end{cases}
\end{aligned}
$$

Note that when $u \leq u^{*}$,

$$
\begin{aligned}
& \{V: 0<V<g(U, W) \mid U=u, W=w\} \\
= & \left\{V: V>0, V^{2} /\left(W^{2}-U^{2}-V^{2}\right)\right. \\
& \left.<g^{2}(U, W) /\left[W^{2}-U^{2}-g^{2}(U, W)\right] \mid U=u, W=w\right\} .
\end{aligned}
$$

By Lukacs' theorem [5], $V^{2} /\left(W^{2}-U^{2}-V^{2}\right)$ is independent of $(U, W)$. Therefore, when $u \in\left[u_{0}, u^{*}\right]$,

$$
\begin{aligned}
& P_{\left(\mu_{1}, 0\right)}\left(A_{0} \mid U=u, W=w\right) \\
= & P_{\left(\mu_{1}, 0\right)}\left(t<V / S<g(U, W) / \sqrt{W^{2}-U^{2}-g^{2}(U, W)} \mid U=u, W=w\right) \\
= & G\left(g(u, w) / \sqrt{w^{2}-u^{2}-g^{2}(u, w)}\right)-G(t) \\
= & G\left(g(u, w) / \sqrt{w^{2}-u^{2}-g^{2}(u, w)}\right)-(1-\alpha),
\end{aligned}
$$

where $G(\cdot)$ is the distribution function of $T$. Define the type I error probability of the LRT, conditioning on $U$ and $W$, as $\alpha_{1}(u, w)=P_{\left(\mu_{1}, 0\right)}\left(A_{0} \mid U=u, W=w\right)$. From (2.7) and (2.8), it follows that

$$
\alpha_{1}(u, w)= \begin{cases}0 & \text { if } 0<u<u_{0}, \\ G\left(g(u, w) / \sqrt{w^{2}-u^{2}-g^{2}(u, w)}\right)-(1-\alpha) & \text { if } u_{0} \leq u<u^{*}, \\ \xi(u, w) & \text { if } u \geq u^{*},\end{cases}
$$

where $\xi(u, w)=P_{\left(\mu_{1}, 0\right)}\left(A_{0} \mid U=u>u^{*}, W=w\right) \leq P_{\left(\mu_{1}, 0\right)}(V / S>t)=\alpha$. Note that $\alpha_{1}(u, w)<\alpha$ for every $(u, w)$ such that $u \in\left(0, u^{*}\right)$, and $\alpha_{1}(u, w) \leq \alpha$ for $u>u^{*}$. To compensate for this shortcoming, we want to find two functions $f_{1}(u, w)$ and $f_{2}(u, w)$ satisfying the following conditions:

(i) If $u \in\left[u_{0}, u^{*}\right)$, then $f_{1}(u, w)<t$ and $f_{2}(u, w)>g(u, w) / \sqrt{w^{2}-u^{2}-g^{2}(u, w)}$;

(ii) $P_{\left(\mu_{1}, 0\right)}\left[f_{1}(U, W)<V / S<f_{2}(U, W) \mid U=u, W=w\right]=\alpha$ for $u \leq u^{*}$.

Clearly, if $f_{1}$ and $f_{2}$ satisfy the above conditions, then $A_{0} \subset\left\{(U, V, S): f_{1}(U, W)\right.$ $\left.<V / S \leq f_{2}(U, W)\right\}$ and $P_{\left(\mu_{1}, 0\right)}\left[f_{1}(U, W)<V / S \leq f_{2}(U, W) \mid U=u, W=w\right]=\alpha$ for $u \leq u^{*}$.

We first consider the case when $u \in\left[u_{0}, u^{*}\right)$. It can be easily verified that the functions $f_{1}$ and $f_{2}$ satisfying the following equations also satisfy the above 
conditions (i) and (ii):

$$
\begin{aligned}
& P_{\left(\mu_{1}, 0\right)}\left(f_{1}<V / S<t \mid U=u, W=w\right)=k\left[\alpha-\alpha_{1}(u, w)\right], \\
& P_{\left(\mu_{1}, 0\right)}\left[g(U, W) / \sqrt{W^{2}-U^{2}-g^{2}(U, W)}<V / S<f_{2} \mid U=u, W=w\right] \\
& \quad=(1-k)\left[\alpha-\alpha_{1}(u, w)\right],
\end{aligned}
$$

where $0<k<1$. From (2.9) and Lukacs' theorem [5], it follows that

$$
G(t)-G\left(f_{1}\right)=k\left[\alpha-\alpha_{1}(u, w)\right] .
$$

This equation may be solved for $f_{1}$ to obtain

$$
f_{1}(u, w)=G^{-1}\left[1-\alpha-k \alpha+k \alpha_{1}(u, w)\right] .
$$

Similarly, (2.10) can be solved to obtain

$$
f_{2}(u, w)=G^{-1}\left\{G\left[g(u, w) / \sqrt{w^{2}-u^{2}-g^{2}(u, w)}\right]+(1-k)\left[\alpha-\alpha_{1}(u, w)\right]\right\} .
$$

Next, we discuss the case when $u \in\left(0, u_{0}\right)$. Following Liu and Berger [4], it is desired to have the line segment joining the origin and the point $\left(u_{0}, v_{0}\right)$ included in the critical region. Note that the equation of this line is $v=\left(v_{0} / u_{0}\right) u$. Therefore, two functions $h_{1}(u, w)$ and $h_{2}(u, w)$ need to be defined that satisfy the following equations:

$$
P_{\left(\mu_{1}, 0\right)}\left(h_{1}<V<v_{0} U / u_{0} \mid U=u, W=w\right)=k\left[\alpha-\alpha_{1}(u, w)\right]=k \alpha,
$$

$$
P_{\left(\mu_{1}, 0\right)}\left(v_{0} U / u_{0}<V<h_{2} \mid U=u, W=w\right)=(1-k)\left[\alpha-\alpha_{1}(u, w)\right]=(1-k) \alpha .
$$

To solve (2.11), note that

$$
\begin{aligned}
& \left\{V: h_{1}<V<v_{0} u / u_{0} \mid U=u, W=w\right\} \\
= & \left\{V: h_{1} / \sqrt{w^{2}-u^{2}-h_{1}^{2}}<V / S<\left(v_{0} u / u_{0}\right) / \sqrt{w^{2}-u^{2}-\left(v_{0} u / u_{0}\right)^{2}}\right\} .
\end{aligned}
$$

Define $f_{1}=h_{1} / \sqrt{w^{2}-u^{2}-h_{1}^{2}}$. In view of (2.13), it is clear that (2.11) can be rewritten as

$$
P_{\left(\mu_{1}, 0\right)}\left[f_{1}<V / S \leq\left(v_{0} U / u_{0}\right) / \sqrt{W^{2}-U^{2}-\left(v_{0} U / u_{0}\right)^{2}} \mid U=u, W=w\right]=k \alpha .
$$

The above equation may be solved to obtain

$$
f_{1}(u, w)=G^{-1}\left\{G\left[\left(v_{0} u / u_{0}\right) / \sqrt{w^{2}-u^{2}-\left(v_{0} u / u_{0}\right)^{2}}\right]-k \alpha\right\} .
$$

Similarly, we can define $f_{2}=h_{2} / \sqrt{w^{2}-u^{2}-h_{2}^{2}}$ and rewrite (2.12) as

$$
P_{\left(\mu_{1}, 0\right)}\left[\left(v_{0} U / u_{0}\right) / \sqrt{W^{2}-U^{2}-\left(v_{0} U / u_{0}\right)^{2}}<V / S \leq f_{2} \mid U=u, W=w\right] .
$$

Equation (2.12) may then be solved to obtain

$$
f_{2}(u, w)=G^{-1}\left\{(1-k) \alpha+G\left[\left(v_{0} u / u_{0}\right) / \sqrt{w^{2}-u^{2}-\left(v_{0} u / u_{0}\right)^{2}}\right]\right\} .
$$


Recall that when $u \geq u^{*}, P_{\left(\mu_{1}, 0\right)}(V / S>t)=\alpha$. Thus, in summary,

$$
\begin{aligned}
& f_{1}(u, w)= \begin{cases}G^{-1}\left\{G\left[\left(v_{0} u / u_{0}\right) / \sqrt{w^{2}-u^{2}-\left(v_{0} u / u_{0}\right)^{2}}\right]-k \alpha\right\} & \text { if } 0<u<u_{0}, \\
G^{-1}\left[1-\alpha-k \alpha+k \alpha_{1}(u, w)\right] & \text { if } u_{0} \leq u<u^{*}, \\
t & \text { if } u \geq u^{*},\end{cases} \\
& f_{2}(u, w) \\
& = \begin{cases}G^{-1}\left\{(1-k) \alpha+G\left[\left(v_{0} u / u_{0}\right) / \sqrt{w^{2}-u^{2}-\left(v_{0} u / u_{0}\right)^{2}}\right]\right\} & \text { if } 0<u<u_{0}, \\
G^{-1}\left\{G\left[\frac{g(u, w)}{\sqrt{w^{2}-u^{2}-g^{2}(u, w)}}\right]+(1-k)\left[\alpha-\alpha_{1}(u, w)\right]\right\} & \text { if } u_{0} \leq u<u^{*}, \\
\infty & \text { if } u \geq u^{*} .\end{cases}
\end{aligned}
$$

Next, recall the definitions of $X, Y, \nu_{1}$, and $\nu_{2}$ in (2.3). The critical region of the LRT can be expressed as $A_{0}=\left\{(U, V, S): X / S>t,(\gamma X+Y) /\left(S \sqrt{1+\gamma^{2}}\right)>t\right\}$. Note that $X, Y$, and $W$ are functions of $(U, V, S)$. Define

$$
\begin{aligned}
& A_{1}=\left\{(U, V, S): f_{1}(U, W)<V / S \leq f_{2}(U, W)\right\} \cap \mathcal{C}, \\
& A_{2}=\left\{(U, V, S): f_{1}(Y, W)<X / S \leq f_{2}(Y, W)\right\} \cap \mathcal{C} .
\end{aligned}
$$

From the above derivation (see (2.9), (2.10), (2.14) and (2.15)), it follows that $P_{\left(\mu_{1}, 0\right)}\left(A_{1} \mid U=u, W=w\right) \leq \alpha$ for all real numbers $u$ and $w>0$. In fact, when $u>0, P_{\left(\mu_{1}, 0\right)}\left(A_{1} \mid U=u, W=w\right)=\alpha$. Similarly, $P_{\left(\gamma \mu_{2}, \mu_{2}\right)}\left(A_{2} \mid Y=y, W=w\right) \leq$ $\alpha$ for all real numbers $y$ and $w>0$. Therefore,

$$
\begin{aligned}
P_{\left(\mu_{1}, 0\right)}\left(A_{1} \cap A_{2}\right) & \leq E\left[P_{\left(\mu_{1}, 0\right)}\left(A_{1} \mid U, W\right)\right] \leq \alpha, \\
P_{\left(\gamma \mu_{2}, \mu_{2}\right)}\left(A_{1} \cap A_{2}\right) & \leq E\left[P_{\left(\gamma \mu_{2}, \mu_{2}\right)}\left(A_{2} \mid Y, W\right)\right] \leq \alpha .
\end{aligned}
$$

Note also that $A_{0} \subset A_{1} \cap A_{2}$ (see conditions (i) and (ii) for $f_{1}$ and $f_{2}$ given above). Thus, by Theorem 2.1, the test with critical region $A_{1} \cap A_{2}$ is a size- $\alpha$ test that is uniformly more powerful than the LRT.

2.1.2 The case of $u_{0}>u^{*}$. In this case we have, similar to (2.6),

$$
\begin{aligned}
& \left\{(U, V, S): V>0, U<u_{0},(U-\gamma V) / S>t \sqrt{1+\gamma^{2}}\right\} \\
= & \left\{(U, V, S): V>0, \gamma V<U<u_{0},(U-\gamma V)^{2} /\left(W^{2}-U^{2}-V^{2}\right)>t^{2}\left(1+\gamma^{2}\right)\right\} \\
= & \left\{(U, V, S): U>u_{0}, V>\gamma U / d+\sqrt{d\left(1+\gamma^{2}\right) t^{2}\left(W^{2}-U^{2}\right)-d U^{2}+\gamma^{2} U^{2}} / d\right\} .
\end{aligned}
$$

Note that the conditional critical region is above the curve

$$
v=\gamma u / d+\sqrt{d\left(1+\gamma^{2}\right) t^{2}\left(w^{2}-u^{2}\right)-d u^{2}+\gamma^{2} u^{2}} / d
$$

(see Figure 2). Let $g(u, w)=\gamma u / d+\sqrt{d\left(1+\gamma^{2}\right) t^{2}\left(w^{2}-u^{2}\right)-d u^{2}+\gamma^{2} u^{2}} / d$ be a function defined for $u^{*}<u \leq u_{0}$. From (2.1) and (2.17), it follows that

$$
A_{0} \cap\left\{(U, V, S): U \leq u_{0}\right\}=\left\{(U, V, S): U \leq u_{0}, V>g(U, W)\right\} .
$$


Consequently, for $u \leq u_{0}$,

$$
\begin{aligned}
P_{\left(\mu_{1}, 0\right)}\left(A_{0} \mid U=u, W=w\right) & \\
= & \begin{cases}0 & \text { if } u \leq u^{*}, \\
P_{\left(\mu_{1}, 0\right)}[V>g(U, W) \mid U=u, W=w] & \text { if } u^{*}<u \leq u_{0} .\end{cases}
\end{aligned}
$$

Note that when $u^{*}<u \leq u_{0}$,

$$
\begin{aligned}
& \{V: V>g(U, W) \mid U=u, W=w\} \\
= & \left\{V: V^{2} /\left(W^{2}-U^{2}-V^{2}\right)>g^{2}(U, W) /\left[W^{2}-U^{2}-g^{2}(U, W)\right] \mid U=u, W=w\right\} .
\end{aligned}
$$

Therefore, again using Lukacs' theorem [5], when $u \in\left(u^{*}, u_{0}\right]$, the type I error probability of the LRT when $\mu_{2}=0$, conditioning on $U$ and $W$, is

$$
\alpha_{1}(u, w)= \begin{cases}0 & \text { if } 0<u \leq u^{*}, \\ 1-G\left(g(u, w) / \sqrt{w^{2}-u^{2}-g^{2}(u, w)}\right) & \text { if } u^{*}<u \leq u_{0}, \\ \xi(u, w) & \text { if } u>u_{0},\end{cases}
$$

where $\xi(u, w)=P_{\left(\mu_{1}, 0\right)}\left(A_{0} \mid U=u>u_{0}, W=w\right) \leq P_{\left(\mu_{1}, 0\right)}(V / S>t)=\alpha$. Clearly, $\alpha_{1}(u, w)<\alpha$ for all $(u, w)$ such that $u \leq u_{0}$, and $\alpha_{1}(u, w) \leq \alpha$ for $u>u_{0}$. To compensate for this shortcoming, we want to find two functions $f_{1}(u, w)$ and $f_{2}(u, w)$ such that

$$
P_{\left(\mu_{1}, 0\right)}\left(f_{1}<V / S \leq f_{2} \mid U=u, W=w\right)=\alpha-\alpha_{1}(u, w),
$$

with the line segment joining the origin and the point $\left(u_{0}, v_{0}\right)$ being contained in the set $\left\{(U, V, S): f_{1}<V / S<f_{2}\right\}$.

To accomplish this, we first define two functions $h_{1}$ and $h_{2}$ by the following two equations:

$$
\begin{aligned}
& P_{\left(\mu_{1}, 0\right)}\left\{h_{1}<V \leq v_{0} U / u_{0} \mid U=u, W=w\right\}=k\left[\alpha-\alpha_{1}(u, w)\right], \\
& P_{\left(\mu_{1}, 0\right)}\left\{v_{0} U / u_{0}<V \leq h_{2} \mid U=u, W=w\right)=(1-k)\left[\alpha-\alpha_{1}(u, w)\right] .
\end{aligned}
$$

Let $f_{i}(u, w)=h_{i}(u, w) / \sqrt{w^{2}-u^{2}-h_{i}^{2}(u, w)}$ for $i=1,2$. The above equations are equivalent to

$$
\begin{aligned}
& P_{\left(\mu_{1}, 0\right)}\left[f_{1}(u, w)<V / S<\left(v_{0} u / u_{0}\right) / \sqrt{w^{2}-u^{2}-\left(v_{0} u / u_{0}\right)^{2}}\right]=k\left[\alpha-\alpha_{1}(u, w)\right], \\
& P_{\left(\mu_{1}, 0\right)}\left[\left(v_{0} u / u_{0}\right) / \sqrt{w^{2}-u^{2}-\left(v_{0} u / u_{0}\right)^{2}}<V / S \leq f_{2}(u, w)\right]=(1-k)\left[\alpha-\alpha_{1}(u, w)\right],
\end{aligned}
$$

since $V / S$ is independent of $(U, W)$.

The above equations may be solved to obtain, for $0<u \leq u_{0}$,

$$
\begin{aligned}
& f_{1}(u, w)=G^{-1}\left\{G\left[\left(v_{0} u / u_{0}\right) / \sqrt{w^{2}-u^{2}-\left(v_{0} u / u_{0}\right)^{2}}\right]-k\left[\alpha-\alpha_{1}(u, w)\right]\right\}, \\
& f_{2}(u, w)=G^{-1}\left\{G\left[\left(v_{0} u / u_{0}\right) / \sqrt{w^{2}-u^{2}-\left(v_{0} u / u_{0}\right)^{2}}\right]+(1-k)\left[\alpha-\alpha_{1}(u, w)\right]\right\},
\end{aligned}
$$

where $\alpha_{1}(u, w)$ is the piecewise-defined function given in (2.19).

Finally, as in Subsection 2.1.1, recall the definitions of $X$ and $Y$ in (2.3) and define

$$
\begin{aligned}
& A_{1}=\left\{(U, V, S): f_{1}(U, W)<V / S \leq f_{2}(U, W)\right\} \cap \mathcal{C}, \\
& A_{2}=\left\{(U, V, S): f_{1}(Y, W)<X / S \leq f_{2}(Y, W)\right\} \cap \mathcal{C} .
\end{aligned}
$$


It follows from the same argument given in Subsection 2.1.1 that the test with critical region $A_{0} \cup\left(A_{1} \cap A_{2}\right)$ is a size- $\alpha$ test that is uniformly more powerful than the LRT.

2.2 Tests for $r>0$. In this section, a test is given that is uniformly more powerful than the LRT when $r>0$. Details of the derivation are omitted here, but they are very similar to those given in Section 2.1. Let $\left(u_{0}, v_{0}\right) \in \mathcal{C}$ be the solution to the following system of equations:

$$
\left\{\begin{array}{l}
(u+r v)^{2}=t^{2}\left(1+r^{2}\right)\left(w^{2}-u^{2}-v^{2}\right), \\
v^{2}=t^{2}\left(w^{2}-u^{2}-v^{2}\right) .
\end{array}\right.
$$

This system can be solved to obtain $u_{0}=c t w / \sqrt{1+t^{2} c^{2}+t^{2}}$ and $v_{0}=u_{0} / c$, where $c=\sqrt{1+r^{2}}-r$. Thus, the equation of the line joining $(0,0)$ and $\left(u_{0}, v_{0}\right)$ is $v=u / c$. Let $d=t^{2}+r^{2}+r^{2} t^{2}, k \in(0,1)$, and

$$
\begin{aligned}
g(u, w) & =\left\{-r u+\sqrt{d\left(1+r^{2}\right) t^{2}\left(w^{2}-u^{2}\right)-d u^{2}+r^{2} u^{2}}\right\} / d, \\
\alpha_{1}(u, w) & =1-G\left(g(u, w) / \sqrt{w^{2}-u^{2}-g^{2}(u, w)}\right), \\
f_{1}(u, w ; k) & =G^{-1}\left\{G\left(u / \sqrt{c^{2} w^{2}-u^{2} c^{2}-u^{2}}\right)-(1-k)\left[\alpha-\alpha_{1}(u, w)\right]\right\}, \\
f_{2}(u, w ; k) & =G^{-1}\left\{G\left(u / \sqrt{c^{2} w^{2}-u^{2} c^{2}-u^{2}}\right)+k\left[\alpha-\alpha_{1}(u, w)\right]\right\} .
\end{aligned}
$$

Note that $u_{0} \equiv u_{0}(w)$ depends on $w$. Define $X$ and $Y$ as in (2.3), and let

$$
\begin{aligned}
& A_{1}=\left\{0<U<u_{0}(W), f_{1}(U, W ; k)<V / S \leq f_{2}(U, W ; k)\right\}, \\
& A_{2}=\left\{0<Y<u_{0}(W), f_{1}(Y, W ; 1-k)<X / S \leq f_{2}(Y, W ; 1-k)\right\} .
\end{aligned}
$$

Then the test with critical region $A_{0} \cup\left(A_{1} \cap A_{2} \cap \mathcal{C}\right)$ is uniformly more powerful than the LRT.

Again, extensions to the general problem (1.1) with $m>2$ and $\Sigma_{0} \neq I$ can be achieved by linear transformation and by applying the intersection-union method described in Section 4 of Liu and Berger [4].

\section{ACKNOWLEDGMENTS}

The authors would like to thank Professor R.L. Berger and an anonymous referee for valuable comments. Y. Wang also acknowledges NIH Grant 1T32CA09667.

\section{REFERENCES}

1. Berger, R. L. (1989), Uniformly more powerful tests for hypotheses concerning linear inequalities and normal means, J. Amer. Statist. Assoc., 84, 192-199. MR 90k:62117

2. Berger, R. L. and Hsu, J. C. (1996), Bioequivalence trials, intersection-union tests, and equivalence confidence sets, Statist. Sci., 11, 283-302. MR 98e:62046

3. Laska, E. M. and Meisner, M. J. (1989), Testing whether an identified treatment is best, Biometrics, 45, 1139-1151. MR 91d:62059

4. Liu, H. and Berger, R. L. (1995), Uniformly more powerful, one-sided tests for hypotheses about linear inequalities, Ann. Statist., 23, 55-72. MR 96m:62108

5. Lukacs, E. (1955), A characterization of the gamma distribution, Ann. Math. Statist., 26, 319-324. MR 16:1034b

6. McDermott, M. P. and Wang, Y. (2001), Construction of uniformly more powerful tests for hypotheses about linear inequalities, J. Statist. Plan. Inf. (to appear). 
7. Sasabuchi, S. (1980), A test of a multivariate normal mean with composite hypotheses determined by linear inequalities, Biometrika, 67, 429-439. MR 82a:62078

8. Sasabuchi, S. (1988), A multivariate test with composite hypotheses determined by linear inequalities when the covariance matrix has an unknown scale factor, Mem. Fac. Sci., Kyushu Univ. Ser. A, 42, 9-19. MR 89k:62075

Schering-Plough Research Institute, 2015 Galloping Hill Road, K-15-2, 2315, KenilWORTh, NEW JERSEY 07033-0539

E-mail address: wayne.wang@spcorp.com

Department of Biostatistics, University of Rochester, 601 Elmwood Avenue, Box 630, Rochester, New York 14642

E-mail address: mikem@bst.rochester.edu 